\title{
A Computational Approach to Color Illusions
}

\author{
Daniele Marini* and Alessandro Rizzi** \\ * Dipartimento di Scienze della Informazione \\ Università degli Studi di Milano \\ Via Comelico 39 - 20135 Milano, Italy \\ marini@eidomatica.dsi.unimi.it \\ ** Dipartimento di Elettronica per l'Automazione \\ Università degli Studi đi Brescia \\ Via Branze $38-25123$ Brescia, Italy \\ rizzi@bsing.ing.unibs.it
}

\begin{abstract}
Tri-stimulus theory of color perception is not able to justify effectively some well known perception phenomena as color illusions and color constancy. Retinex theory, by Land and McCann, grounds color perception on a color space based on three lightness computed as relative reflectance along multiple exploration paths of the perceived scene. This paper considers in a new light Retinex theory, as a theory which tries to justify not only color constancy but also illusions arising from simultaneous contrast configurations. An improvement to Retinex computational model is presented in the paper, which selects Retinex computation paths by approximating a brownian path. The algorithm has been tested not only on traditional Mondrian patches, but also on natural pictures and photographs and on typical color illusion patches. The examples demonstrate the ability of the model to emulate human color perception behavior.
\end{abstract}

\section{$1 \quad$ Introduction}

Some common visual experiences show that human color perception cannot be completely explained by recurring to tri-stimulus theory, which dates back to von Helmholtz and Young. Let us consider a picture taken with a photo camera in an interior illuminated by tungsten lamps: a dominant red-orange modifies the original colors. The same happens when we take some shot using a TV camcorder, without "balancing the white". If, on the contrary, we observe an interior scene in different illumination condition, e.g. illuminated by a tungsten lamp or by the sunlight from a window, we do not perceive such deep differences.

This problem is known as color constancy, and the human ability to compensate varying light conditions cannot be explained by recurring to a color theory based on a physical model of light-matter interaction and on the tri-stimulus theory. Indeed such theories can explain how the spectral composition of light reflected by a given and known surface can change when the spectral composition of the illumination changes. 
Many physiological experiments and analysis have shown that human visual system is grounded on the stimulation of cones by three fundamental ranges of frequencies, so that all experts agree in the assumption that cones have three different pigments that allow them to react differently to these three light frequency ranges. What tristimulus theory is not able to explain is how such triple stimulus is elaborated by the human visual system, so that color perception, in large part, is invariant to varying illumination conditions.

Color constancy is usually considered as the ability to perceive the same color in varying viewing conditions. Brainard \& Wandell formalize the problem of color constancy, for a visual configuration typical of the first Land's experiments. Let a surface composed of different matte materials (reflecting by Lambert law) be given; an observer looks at the surface, he has three classes of photoreceptors. The spectral power distribution of the ambient light is $E\left(\lambda_{n}\right)$, where $\lambda_{n}$ is given at discrete wavelengths values $n=1, . ., N$. Let $S^{x}\left(\lambda_{n}\right)$ be the reflectance of the surface at a point $x$. The light arriving at the observer's eye is the color signal, and it is given by:

$$
C^{x}\left(\lambda_{n}\right)=E\left(\lambda_{n}\right) S^{x}\left(\lambda_{n}\right)
$$

The observer has three arrays of photoreceptors that spatially sample the color signal; their response is computed from the color signal and the spectral sensitivity of the photoreceptor's pigment in the $\mathrm{k}$-th class $R_{k}\left(\lambda_{n}\right)$ ( $\mathrm{k}$ is three as the retinal cone pigments):

$$
\rho_{k}^{x}=\Sigma_{n=1 . . N}\left(C^{x}\left(\lambda_{n}\right) R_{k}\left(\lambda_{n}\right)\right)=\Sigma_{n=1 . . N} E\left(\lambda_{n}\right) S^{x}\left(\lambda_{n}\right) R_{k}\left(\lambda_{n}\right)
$$

The equation can be written in matrix form:

$$
\rho^{\mathrm{x}}=\Lambda_{E} \sigma^{\mathrm{x}}
$$

where $\Lambda_{E}$ is a $3 \times N$ matrix and each entry is $E\left(\lambda_{n}\right) R_{k}\left(\lambda_{n}\right)$, i.e. it depends only from the spectral power distribution of the ambient light and on the receptor spectral sensitivity. $\Lambda_{E}$ is also called lighting matrix.

$\rho^{X}$ is a vector of 3 components, representing the response of the three receptor classes, at location $x$.

$\sigma^{X}$ is the surface spectral reflectance at point $x$, an $\mathrm{N}$-vector.

The problem of color constancy can now be stated: if the receptor response vector is given, and also given are $E\left(\lambda_{n}\right)$ and $R_{k}\left(\lambda_{n}\right)$ (therefore if the lighting matrix is fully known), to determine the vector $\sigma^{\mathrm{X}}$ in any point $\mathrm{x}$ of the surface it is necessary to invert the linear equation (1). The system has a unique solution if the lighting matrix is square, i.e. if only three wavelength samples are taken, otherwise it has infinite solutions.

More complex is the problem when the ambient light spectral power distribution is not known and it must be estimated from the given parameters, or when a light source spectral distribution is changing in time. Brainard and Wandell discuss under what conditions the ambient light coefficient can be estimated so that the problem can be reduced to the solution of a linear system. In any case more information is required 
at this aim; in practical applications, like color balancing in video cameras, a reference surface is taken, to which all other colors are normalized.

Based on the above discussion the problem arises of understanding how the human visual system can reconstruct the missing information of light source spectral composition. What we assume, following hypothesis first proposed by E. Land, is that the color perception is the result of a complex comparison process among different visual areas and colored patches. The input to this process is the stimulus produced by cones and rods in the retina, and the process is completed in the higher cortical visual area of the brain.

\section{Retinex color theory}

Retinex theory, due to Edwin Land, assumes that color perception depends strictly on the neural structure of human visual system; being not clear if the retina or the cortex plays the central role, Land coined the term "Retinex" derived from retina and cortex.

The fundamental observation that drove Edwin Land in Retinex theory development is: ".. the eye, in determining color, never perceives the extra red [produced by a tungsten lamp] because [the color] does not depend on the flux of radiant energy reaching it." The first Land's experiment was based on taking two black and white slides with a red and green filter, and projecting them through the same filters: what appears, counter-intuitively, is an image having all the original colors!

Following other experiments, Land defined a quantity, named lightness, which is associated to every object of a scene, and which does not change as illumination conditions and object's location (closer or fartherfrom other objects with different lightness) change. Lightness is perceived by human visual system independently from the light flux that impinges the eye. With a second experiment, Land and McCann verified that a full color sensation of color gamut can be obtained producing a light stimulus to rods and long wavelength (red) sensitive cones: color sensation is again non depending from spectral fluxes reflected by the single objects, but from a comparison of lightnesses. A third experiment demonstrated that color sensation produced by the so called Mondrian, is still independent from reflected spectral flux, or more properly from the product of spectral reflectivity by incident light energy produced by a light source, which is the property of color constancy.

Land and McCann defined a measurable physical quantity that can be correlated to the lightness: it is the ratio between the integrated radiance from a Mondrian area and the integrated radiance from a white paper measured under white illumination, with three filters that perform as cone spectral sensitivity. The color sensation is produced by a comparison of the three resulting values, even if, as Land says, no corresponding neural regions can be exactly identified in human visual system. The three numbers characterize a measure of lightness, allowing to propose a color geometric space, that can be used to measure and compare colors and color scales. Moreover the experiments show that the color sensation is not due only to the spectral characteristics of the light impinging the eye, because in that case the color of the same patch illuminated by two different light sources should be perceived of two different colors. We recall that this effect can indeed be perceived in "void condition", i.e. when the illuminated patch is isolated and the observer has no other stimulus from the surround. The Retinex theory assumes that human vision is based on three retinal-cortical system, each processing independently the low, middle and high frequency of the visible spectrum. 
Each system forms a separate image of the world; the images are not mixed but compared and each system discovers independently the reflectance of the various region of the image, independently also from variation of the light source spectrum.

Land \& McCann discuss how the relative reflectance computation can be simulated by an electronic equipment and by a neural net. They arrive at the conclusion that a couple of excitatory and inhibitory neurons in a chain can account for the reset mechanism required by the search for the lightest area (i.e. the white).

The theory assumes that the three Retinex systems receive their stimulus by the retinal cones, that have an absorption non uniform in their wavelength range sensitivity. So what is effectively fed into the computation cycle is the integral of the spectral product of absorbency of the cone pigments times the irradiance times the reflectance of the patch.

\section{A computational implementation of Retinex theory}

The relative reflectance of a colored patch is the mean value of relative reflectance computed along a number $\mathrm{N}$ of random paths to that patch:

$$
R^{\Lambda}(i)=\frac{\sum_{j=1}^{N} R^{\Lambda}(i, j)}{N}
$$

where $\Lambda$ is the low, middle or high wavelength range and:

$$
R^{\Lambda}(i, j)=\sum_{k} \delta \log \frac{I_{K+1}}{I_{k}}
$$

is the reflectance of a colored patch i relative to a patch $\mathrm{j}$, and:

$$
\delta \log \frac{I_{K+1}}{I_{k}}=\left\{\begin{array}{c}
\log \frac{I_{K+1}}{I_{k}} \text { if }\left|\log \frac{I_{K+1}}{I_{k}}\right|>\text { threshold } \\
\text { Oif }\left|\log \frac{I_{K+1}}{I_{k}}\right|<\text { threshold }
\end{array}\right.
$$

$I_{k}$ is the intensity in location $\mathrm{k}$ of the image, and should be computed, for a given wavelength range $\Lambda$, as:

$$
I_{k}=\int_{\lambda \in(400,700)} a^{\Lambda}(\lambda) L(\lambda) \rho(\lambda) d \lambda
$$

where: $a^{\Lambda}(\lambda)$ is the absorbency of the cone pigment for the range $\Lambda, L(\lambda)$ is the spectral irradiance on the patch and $\rho(\lambda)$ is its spectral reflectivity.

These computations are to be executed for the three fundamental wavelengths range $\Lambda$, corresponding to low, middle and high bands. 
The above model depends on the randomness and number of paths that are chosen for the computation of the relative reflectance, moreover it depends on the value of the threshold that makes less relevant low reflectance ratios, that correspond to smooth change in color due to non uniform illumination. The basic Land algorithm, moreover, has a reset mechanism: if during a path computation a lighter area is found the cumulated relative reflectance is forced to 1 , making the average computation to restart form this area. The effect of the reset mechanism is to consider the lightest area of an image as the reference value of the color white.

A critical problem in the above algorithm is the choice of the random path. An analysis of this problem has allowed us to propose a new solution, based on the Brownian motion. This assumption derives from results inresearch on human cortical visual system, where the distribution of the receptive fields of area V4 (which is considered the most responsive to color signal, see: Zeki) shows a Brownian path aspect.

\subsection{The Brownian-path algorithm}

To implement the Brownian motion to approximate a path in the Retinex Algorithm we have adopted the random mid point displacement technique (see: Saupe). The number of recursions to generate the brownian path is a power of 2 . During the path traversal the algorithm scan converts the pixels computing the relative reflectance for each pixel along the edge:

foreach chromatic channel compute sequentially each pixel as follow: generate $N$ random points in the image and foreach random point:

generate a path by $2^{\wedge} M$ random mid-point displacement;

follow the generated path and foreach pixel calculate the new chain function value (Knew);

if Knew >threshold then Kold=Knew;

if Knew>1 then Kold=1; (a pixel with an higher lightness value has been visited)

newChromaticPixelValue $=$ average of all $N$ final values Kold;

(I foreach random path)

Comparing results with this algorithm to the traditional random path generation approach, we have observed a significant reduction of the number of paths necessary to approximate the lightness value of each pixel. While the random-path algorithm requires about 200 paths, the brownian-path algorithm gives very good results with 20 or sometimes even 4 paths per pixel. So the time complexity spent in generating the brownian path approximation and scan conversion of path edges is improved by the drastic reduction of the total number of paths.

\section{Retinex application on color illusions}

Besides color constancy, also colour illusions are difficult to explain with classical color models. A well known color illusion derives from what Itten, Albers and others call simultaneous contrast, which arises when a small grey square lays over a larger 
and saturated background color. In this case what the observer perceives is not the grey but a hue which tends to the complemetary color of the background. We have applied the Retinex algorithm to some classical color patches and the results have shown that the Retinex algorithm computes a color triplet which is vey much alike the perceived color in natural conditions.

In Color figure 1 a color illusion by $\mathbf{J}$. Albers is simulated. The configuration shows the effect on the human vision of simultaneous contrast of different color patches. In this example we perceive two different brown patches, the top one with a higher saturation then the bottom one. A similar illusion arises when a gray small square is put on a bigger colored background (see Itten); in this case the perceived color is much more alike the complementary of the background (see Color figure 2). Both examples have been computed using the brownian path algorithm with 20 paths.

In color figure 3 another illusion by Joseph Albers is simulated with Retinex algorithm: the gray background is perceived as non uniform and smoothly changing in an opposite way as the internal squares. The Retinex simulation, using the random path algorithm with 20 paths, shows this effect.

In color figure 4 the Retinex algorithm has been applied to a photograph to equalize colors, using brownian motion approximation on 20 paths.

\section{Conclusions and perspectives}

We have implemented two algorithms that compute the Retinex model of color vision proposed by Edwin Land in late ' 60 . The algorithms differ in the technique adopted for choosing the paths necessary to explore the image to compute the three Retinex lightness. The first algorithm selects the paths in a pseudo-random way, while the second approach adopts an approximation of a Brownian path by mid-point displacement. The main criticism against Retinex theory concerns its inability to cope with situation with varying backgrounds, therefore reducing predicatability of the results. We consider that this is more a confirmation of the theory rather then a defect: the model emulates human behavior in color perception which is frequently faked by simultaneous contrast effects, so that the perceived color is different form the measured one in a controlled context. This behavior has been simulated in a variety of examples, trypical of color illusions.

Future developments of this research will modify the algorithm to filter digitized images by computing first an approximation of human cones response before the application of the Retinex algorithm. Moreover computational aspects will be explored to improve algorithm efficiency, that in typical images has a computation time ranging from few minutes to half an hour.

\section{References}

[1] Albers J. Interaction of Color, Yale University Press, New Haven (1975)

[2] Brainard D.H. and Wandell B.A., "Analysis of the Retinex theory of color vision", JOSA-A, 3(10), 1651-1661 (1986)

[3] D'Zmura M., Lennie P. "Mechanisms of Color Constancy", Journal of Optical Society of America, 3,10, 1662-1672 (1986)

[4] Itten J. Kunst der Farbe, Otto Meier Verlag, Ravensburg (1970) 
[5] Judd. D.B. "Appraisal of Land's work on two-primary color projections", Joumal of Optical Society of America 50, 254-268 (1960)

[6] Land E. and McCann J., "Lightness and Retinex Theory", Journal of Optical Society of America, 61,1, 1-11 (1971)

[7] Land E., "Recent Advances in Retinex Theory and Some Implications for Cortical Computations: Color Vision and the Natural image", Proc. Natl. Acad. Sci. USA, Vol. 80, 5163-5169 (1983)

[8] Land E., "The Retinex Theory of Color Vision", Scientific American, 237, 3, 217 (1977)

[9] Landy M.S., Movshon J.A. Ed.s Computational Models of Visual Processing, The MIT Press, Boston (1991)

[10]Luong Quang-Tuan, "La Couleur en Vision par Ordinateur: 1. une Revue", Rapports de Recherche INRIA, n. 1251 (1990)

[11]Maloney L.T., Wandell R.A. "Color Constancy: A Method for Recovering Surface Spectral Reflectance", Journal of Optical Society of America, 3,1, 29-33 (1986)

[12]McCann J.J. \& Houston K.L., "Calculating Colour Sensation from Arrays of Physical Stimuli", IEEE Transaction on SMC, SMC-13, 5, 1000-1007 (1983)

[13]Saupe D. "Algorithms for random fractals" in: Barnsley et alt. The Science of Fractal Images, Springer Verlag, New York (1988) IEEE Computer Graphics \& Applications, November (1993)

[14]von Helmholtz, H., Optique physiologique, Paris, Edition Jacques Gabay, trad. par Javal E. et Klein N.Th. (1989)

[15]Wandell B.A., Fundations of Vision, Sinauer Associates Inc. Publishers, Sunderland, Massachusetts (1995)

[16]Wyszecky G., Stiles W.S., Color Science: Concepts and Methods, Quantitative Data and Formulas, J. Wiley \& Sons, New York (1982) the II Annual Bionics Symposium, Vol.1, 126-141 (1961)

[17]Zeki S. A Vision of the Brain, Blackwell Scientific Pub., Oxford (1993)

\section{Color Figures}

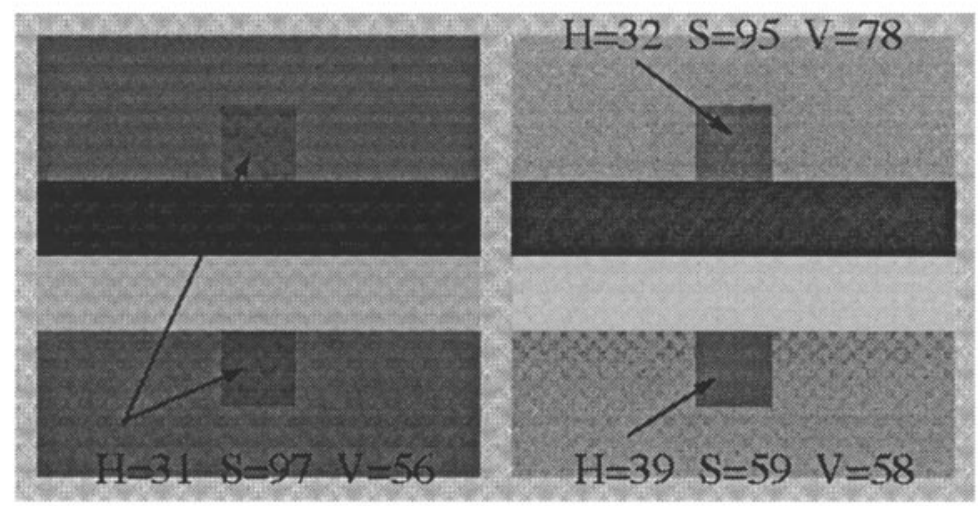

Color Figure 1 - An illusion by Josef Albers 

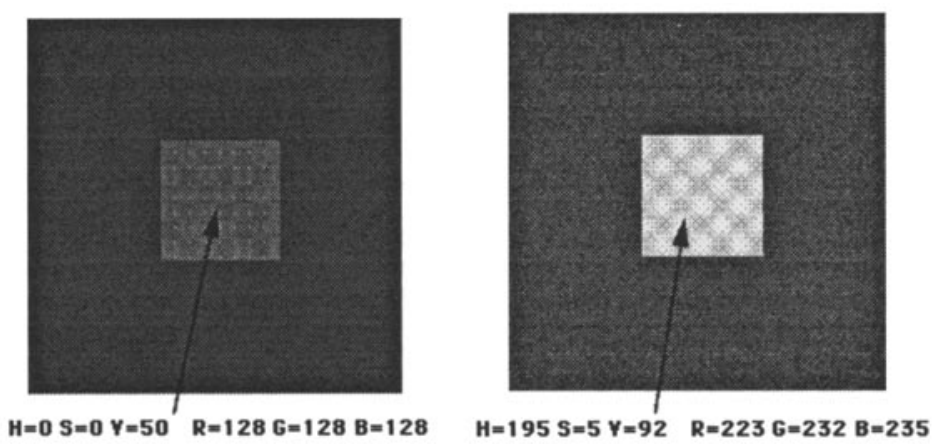

Color Figure 2 - The gray foreground is perceived as of the complementary color of the background
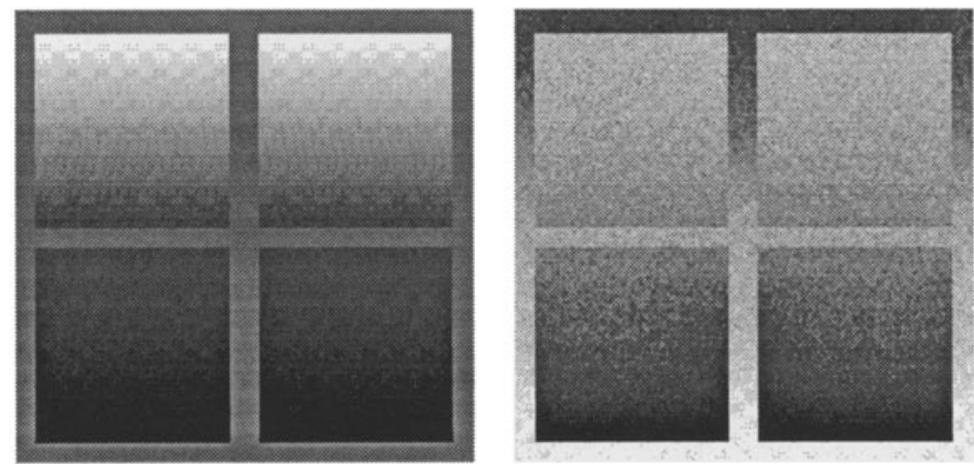

Color Figure 3 - Another illusion by Joseph Albers: left original image, right Retinex filtered image..
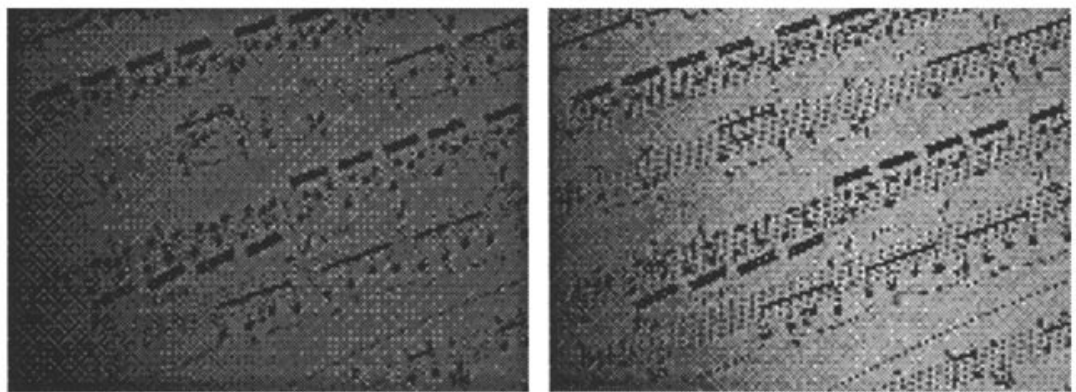

Color Figure 4 - The effect of Retinex algorithm in a non uniform illumination with brown dominant 